Buendía Eisman, Leonor; Expósito López, Jorge; Sánchez Martín, Micaela (2012). Investigación evaluativa de programas de formación profesional para el empleo en el ámbito local. Revista de Investigación Educativa, 30 (1), $161-179$.

\title{
INVESTIGACIÓN EVALUATIVA DE PROGRAMAS DE FORMACIÓN PROFESIONAL PARA EL EMPLEO EN EL ÁMBITO LOCAL
}

\author{
Leonor Buendía Eisman' \\ Jorge Expósito López \\ Micaela Sánchez Martín
}

\section{RESUMEN}

En el presente artículo se presentan los principales aspectos metodológicos de una investigación realizada con objeto de evaluar los programas de Formación Profesional para el Empleo desarrollados en la Comarca de Alhama de Granada, estos son: diseño de la evaluación, procedimiento de recogida de datos, población y muestra, técnicas e instrumentos para la recogida de datos y técnicas de análisis de datos. Los principales objetivos de la evaluación se centran en analizar las necesidades de Formación Profesional para el Empleo, identificar los Nuevos Yacimientos de Empleo, evaluar la satisfacción con los cursos realizados durante dicho periodo. Para realizar un estudio comparativo entre las necesidades formativas, la formación ofertada y el impacto de está sobre la realidad socioeconómica de la zona objeto de estudio.

Palabras clave: Investigación evaluativa, evaluación de programas, metodología, formación profesional para el empleo, inserción laboral.

1 Leonor Buendía Eisman es Catedrática del Área MIDE de la Facultad de Ciencias de la Educación de la Universidad del Granada, lbuendia@ugr.es; Jorge Expósito López es Profesor Asociado del Área MIDE de la Facultad de Ciencias de la Educación de la Universidad de Granada, jorgeel@ugr.es

Dirección: Campus Universitario Cartuja, s/n, 18071, Granada.

Micaela Sánchez Martín es Profesora Asociada del Área MIDE de la Facultad de Educación de la Universidad de Murcia, micaelasmartin@um.es.

Dirección: Campus Espinardo, 30100, Murcia. 


\title{
EVALUATION RESEARCH OF PROFESSIONAL TRAINING PROGRAMMES APPLIED TO LOCAL EMPLOYMENT
}

\begin{abstract}
The present study presents the main methodological features of a research study focused on the assessment of Vocational Training Programmes carried out in the region of Alhama de Granada (Spain). These features are: evaluation design, data collection procedures, population and sample, data collection instruments, and data analysis procedures. The main aims of our assessment focused on discussing the needs for Vocational.Training in this geographical area, identifying new professional niches where people may be employed, and assessing users' satisfaction with training courses completed during a period of time. We also intend to carry out a comparative study between training needs, training offered and its impact on the socioeconomic situation of the area under study.
\end{abstract}

Keywords: Evaluation research, programme assessment, methodology, vocational training programmes, professional integration services.

\section{INTRODUCCIÓN}

Actualmente las zonas rurales continúan presentando unos estándares educacionales y económicos inferiores a las áreas urbanas (Albrecht et al, 2000; García, 1999). El escaso y desigual desarrollo socioeconómico de las áreas rurales ha originado una informalización del trabajo y ha producido una disparidad en la distribución de empleos e ingresos entre las comunidades rurales (García e Izcara, 2000).

Los conceptos desarrollo local, empleo y exclusión social están conectados entre si, ya que dependen de las mismas dimensiones políticas sociales y económicas. La existencia de colectivos de especial dificultad, excluidos socialmente no por el mero hecho de estar desempleados, sino por carecer de expectativas de futuro, como son jóvenes, mujeres, inmigrantes y personas con diversidad funcional, supone una mayor concreción de actuaciones más centradas en el ámbito local. En esta línea se trata de potenciar los Nuevos Yacimientos de Empleo, en adelante NYE, dado que se contemplan como una de las políticas activas más destacadas en la creación de empleo y una de las mayores opciones para la empleabilidad de estos colectivos.

Con este estudio se pretende realizar un diagnóstico de necesidades de Formación Profesional para el Empleo (FPE) en la Comarca de Alhama de Granada, prestando especial atención a colectivos con especiales dificultades de inserción, mediante la evaluación de la adecuación de los programas de formación que se han venido realizando y el análisis de nuevas necesidades formativas para mejorar la capacidad de gestión y la renovación de los sistemas de formación. La finalidad del estudio es principalmente práctica puesto que pretendemos realizar propuestas de intervención y mejora para la integración y desarrollo de los NYE e implementar nuevas líneas de intervención y formación, adaptadas a estos nuevos empleos. 


\section{CONTEXTO DEL ESTUDIO}

La principal y más influyente característica del territorio objeto de estudio en lo que respecta al mercado de trabajo, es que se trata de una zona rural, lo que supone unas oportunidades de empleo limitadas a la agricultura y a los comercios familiares que junto con las pequeñas empresas o microempresas suponen el nicho de empleo predominante en la zona. El motor básico de la economía es el sector servicios que ocupa al 43\% de la población (Instituto de Estadística de Andalucía, 2007), sin embargo, existe una gran escasez de personal cualificado. Las precarias condiciones laborales que el empresario ofrece, hace casi imposible que se cubran las ofertas. Este desajuste entre la oferta y la demanda de empleo supone un desaprovechamiento de los recursos humanos de la comarca, particularmente de los jóvenes que se ven forzados a buscar oportunidades de trabajo fuera de su territorio, produciéndose una pérdida poblacional debido a la caída de la natalidad y a los movimientos migratorios, lo que conlleva un envejecimiento de la población que se traduce en un estancamiento del nivel de desarrollo.

Esta zona está formada por municipios muy dispersos y con escasas comunicaciones con el resto de la provincia, presentando la mayoría de la población escasa disponibilidad para viajar. En estas condiciones la formación se reduce a la oferta formativa que se imparte en Alhama, y esta no siempre se ajusta a las necesidades demandadas por los posibles trabajos de la zona. Cabe destacar también la existencia de una cultura subsidiaria muy arraigada, acompañada de actitudes resignadas y conformistas por lo que el porcentaje de iniciativas empresariales es bajo. Esto, unido al continuo cambio que se está produciendo en el mercado de trabajo, hace necesario disponer de diseños formativos ajustado a las necesidades y posibilidades de la población que faciliten la inserción laboral de sus habitantes.

En el Libro Blanco de Delors (1993), aparece la definición de NYE como una estrategia para luchar contra el paro estructural y como una oportunidad de intervención en la que participan conjuntamente los agentes públicos, sociales y privados, en un marco de concreción social, con el objetivo de reducir el desempleo a partir de la satisfacción de unas carencias sociales que hay que resolver. La fórmula propuesta es ir al encuentro de las nuevas necesidades y darles respuesta mediante iniciativas locales de desarrollo, estimulando así la creación de empleo estable y de calidad. Se trata en definitiva de hacer emerger una serie de ocupaciones inexistentes o incipientes superando los obstáculos que impiden su desarrollo normal.

Las iniciativas locales se dedican a construir un entorno a partir de las singularidades y de las riquezas de cada territorio, combinando las dimensiones económicas y sociales. La formación para el trabajo en el contexto rural tiene como principal característica su vinculación con las estrategias de desarrollo local que, "entre otras consecuencias, tienen la de contribuir a fijar la población al territorio" (Manzanares 2006: 173). Las nuevas ocupaciones encuentran su mejor expresión si se abordan desde la óptica de las iniciativas locales de desarrollo y empleo, siendo requisito necesario para la superación de los obstáculos y el estimulo de los nuevos empleos la fuerte colaboración y la corresponsabilidad entre distintas administraciones, agentes sociales y económicos a escala territorial, realizando un trabajo en cascada. Mediante esa cooperación es importante 
intentar crear un clima social que estimule la implicación activa de todos los agentes sociales y de la población en general, la búsqueda de un desarrollo sostenible, la lucha contra la exclusión, y la satisfacción de las necesidades de la población.

Los NYE deben formar parte de las políticas de desarrollo local, ya que entre sus propósitos básicos destacan la creación y consolidación de un tejido empresarial propio que genere abundante empleo estable y consecuentemente crecimiento económico, con el fin último de aumentar el bienestar de la población.

\section{OBJETIVOS}

En esta investigación nos proponemos los siguientes objetivos:

1. Analizar las necesidades de FPE en la comarca de Alhama de Granada.

2. Evaluar los cursos desarrollados y al profesorado que los ha impartido.

3. Realizar un estudio comparativo entre las necesidades de FPE, la formación ofertada y el impacto de ésta sobre la realidad socioeconómica de la comarca de Alhama de Granada.

4. Identificar los NYE, en la Comarca de Alhama de Granada, para el establecimiento de nuevos procesos formativos.

\section{MÉTODO}

Los modelos de evaluación de programas tradicionalmente se han centrado en la evaluación de los resultados finales, sin estudiar los procesos ni plantearse mejoras (Expósito; Olmedo y Fernández, 2004). En los últimos años estos aspectos han sido considerados en la evaluación de programas formativos, integrando metodologías cualitativas y cuantitativas (Berrocal y Buendía, 2003; Chiva, 2006; Manzanares, 2006; Pérez Juste 2000) según los objetivos que se pretendían cubrir. La mayoría de ellos iban dirigidos a la mejora de programas y a la evaluación de las tasas de inserción profesional, centrándose en colectivos en riesgo de exclusión social.

Los modelos de evaluación del impacto son considerados por diferentes autores (Biencinto y Carballo, 2004; Folgueiras y Marín, 2009; Tejada, 2008), una estrategia de la propia evaluación de la acción formativa, más allá del momento y el espacio de la misma; de hecho, en los modelos de evaluación del impacto (evaluación Respondente de Stake, 1976) o el Modelo CIPP de Stufflebeam y Shinkfield, 1987), se considera la pertinencia e idoneidad de la evaluación diagnóstica, su ajuste a necesidades y el dispositivo inicial de la formación. Estos modelos aportan información del proceso, realizándose una evaluación formativa, y de los resultados o fase final de la evaluación. El ciclo de la vida del programa abre distintas posibilidades en cuanto al momento de realizar la evaluación. Al tratar la evaluación de impacto es importante indicar el momento en la realización de la misma. Un esquema lo presentamos en la siguiente imagen.

Debido a la complejidad del mundo de la formación para el empleo y de los objetivos establecidos, nosotros hemos optado por un modelo de evaluación que se centra tanto en los procesos como en los resultados (de Miguel y otros, 2008; Buendía y Berrocal, 2008; Perales, Jornet y Suarez, 2000), con un diseño abierto elaborado para este estudio. 


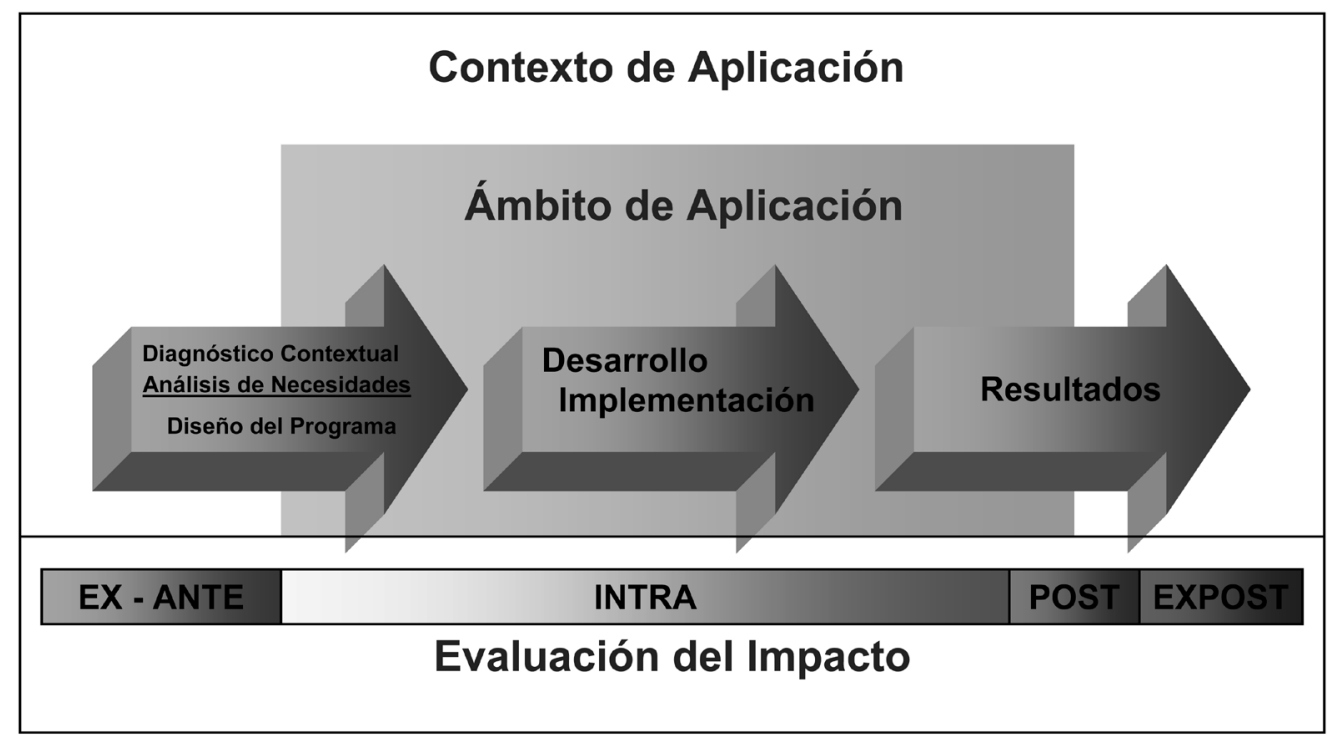

FIGURA 1

EVALUACIÓN DEL IMPACTO DE LOS GRUPOS DE FORMACIÓN PROFESIONAL PARA EL EMPLEO

En consecuencia se ha asumido la complementariedad metodológica, conjugando el uso de un enfoque cuantitativo, más adecuado para medir efectos finales y cuantificables; con el enfoque cualitativo, más apropiado para la interpretación de los significados y la obtención de visiones generales más profundas, con objeto de mejorar el desarrollo y la implementación de los programas. Partimos de un estudio exploratorio para el análisis del contexto y un estudio analítico en profundidad para constatar la adecuación de los programas formativos ofertados a las necesidades de la zona y a los posibles nichos laborales. Igualmente en esta segunda fase evaluamos la satisfacción con la formación recibida y la inserción laboral de los beneficiarios de las acciones formativas.

\section{I. Diseño de evaluación}

El diseño o plan de acción seguido en esta investigación, la presentamos en la tabla 1.

Para realizar la evaluación de impacto, según las acciones formativas, elegimos los siguientes momentos:

- Antes de la ejecución de los distintos programas formativos realizamos un análisis documental del contexto socioeconómico.

- El momento de la evaluación es diferente en cada Centro. En los Talleres de Empleo se realiza al finalizar el programa. Evaluamos las actividades del proceso identificando aciertos, errores y dificultades. Igualmente en el curso de Informador Turístico se realiza la evaluación una vez finalizada la acción formativa; sin embargo en la Escuela Taller se realiza la evaluación durante el desarrollo del proceso. 


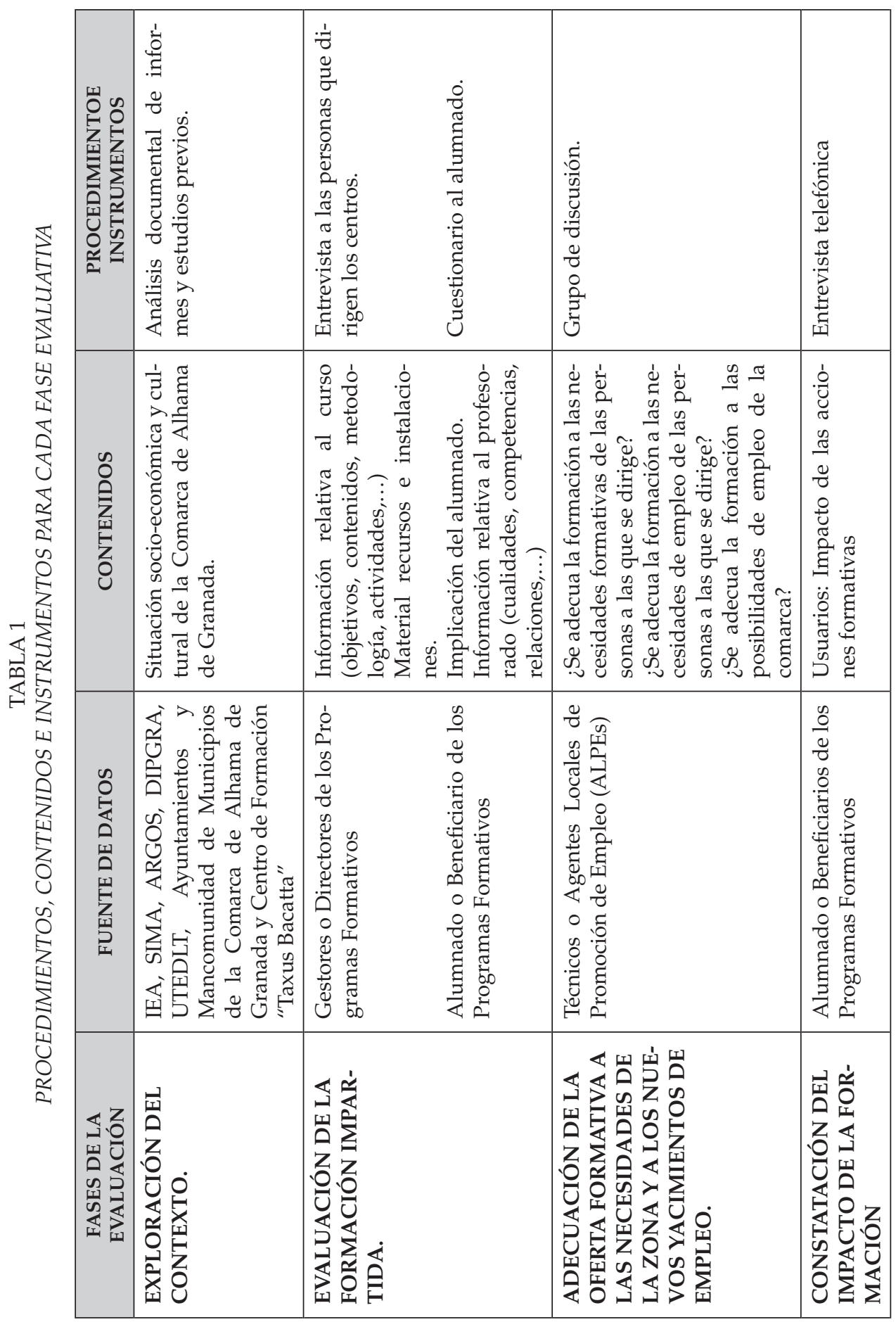

RIE, vol. 30-1 (2012) 
- Al finalizar el proceso formativo de los Talleres de Empleo realizamos un grupo de discusión con los Agentes Locales de Promoción de Empleo (ALPEs); y seis meses después de la ejecución, mediante entrevista telefónica, evaluamos la satisfacción de los beneficiarios, los niveles de inserción y las nuevas demandas formativas.

El acceso a los escenarios viene marcado por las pautas de la planificación del proceso evaluativo, como muestra la tabla $\mathrm{n}^{\circ} 2$. En esta planificación temporal diferenciamos los momentos siguientes de acceso a los escenarios:

1. Se requiere información a los distintos Ayuntamientos, Mancomunidad de Municipios de la Comarca de Alhama, Diputación de Granada y diversos organismos oficiales.

2. Toma de contacto con los directores de las acciones formativas, para informar y establecer las condiciones del contrato comunicativo de las entrevistas, las cuales tuvieron lugar en sus respectivos centros de trabajo.

3. Tras la solicitud y concesión de los permisos oportunos la investigadora se traslada a los respectivos centros de formación para pasar los cuestionarios al alumnado. Cabe destacar una excepción y es que el curso de informador turístico ha finalizado y hay que localizar a las alumnas de forma individual.

4. Solicitud de permiso a la directora de la Unidad Territorial de Empleo Desarrollo Local y Tecnológico (UTEDLT) de Alhama de Granada para realizar un grupo de discusión en su sede con los ALPEs, con los que se mantuvieron varios contactos antes de la cita para tratar de asegurarnos su presencia en el grupo.

5. Transcurridos seis meses desde la finalización del curso se contacta con el alumnado vía telefónica para la realización de la entrevista. Como se puede observar en la tabla $\mathrm{n}^{\circ} 2$, el proceso se inicia con el análisis de fuentes documentales y

TABLA 2

PLANIFICACIÓN DEL PROCESO EVALUATIVO

\begin{tabular}{|c|c|}
\hline $\begin{array}{l}\text { F } \\
\text { A } \\
\text { S }\end{array}$ & $\begin{array}{l}\text { Información bibliográfica general y revisión de documentos sobre detec- } \\
\text { ción de necesidades de formación e impacto de los procesos formativos y } \\
\text { NYE. }\end{array}$ \\
\hline $\begin{array}{l}\text { E } \\
1\end{array}$ & $\begin{array}{l}\text { Análisis socioeconómico y documental sobre las necesidades de formación } \\
\text { de la zona. }\end{array}$ \\
\hline $\begin{array}{l}\text { F } \\
\text { A }\end{array}$ & $\begin{array}{l}\text { Información bibliográfica general y revisión de documentos sobre detec- } \\
\text { ción de necesidades de formación e impacto de los procesos formativos }\end{array}$ \\
\hline $\begin{array}{l}S \\
F\end{array}$ & Recogida de información: Directores/as y alumnos/as. \\
\hline 2 & Análisis de datos \\
\hline $\mathbf{F}$ & $\begin{array}{l}\text { Recogida de información: Grupo de Discusión con los ALPEs. } \\
\text { Análisis de datos }\end{array}$ \\
\hline $\begin{array}{l}S \\
E\end{array}$ & $\begin{array}{l}\text { Recogida de información: Entrevista telefónica. } \\
\text { Análisis de datos. }\end{array}$ \\
\hline 3 & Elaboración de informe final. \\
\hline
\end{tabular}


finaliza con la elaboración del informe, variando los procedimientos de recogida de datos e informaciones en función del tipo de información requerida para dar respuesta a los objetivos del estudio.

\subsection{Procedimientos de recogida de información}

- Cuestionario a los beneficiarios de las acciones formativas (alumnado) para conocer la satisfacción con la formación recibida empleamos el cuestionario desarrollado por Buendía y Berrocal (2008) para la evaluación de los cursos del Proyecto ACERCA. Mediante el uso de este instrumento se pretende obtener información relativa al curso: objetivos, contenidos, metodología, actividades, material, recursos e instalaciones, implicación por parte del alumnado, así como información relativa al profesorado: cualidades, competencias, relaciones.

- Entrevista a los gestores de los programas formativos para conocer la satisfacción con la formación impartida y entrevista telefónica a los beneficiarios para constatar el impacto de los programas formativos. Elegimos la entrevista personal como forma de recoger la información entre los implementadores y los beneficiarios de las acciones formativas con la finalidad de evaluar los cursos de formación y su impacto socioeconómico, por considerarla la forma más completa de recoger la información. Concretamente adoptamos la entrevista semiestructurada, en la que se prepararon una serie de preguntas para guiar la conversación. A la realización de la entrevista le precede la fase de preparación (Yaus, 1990) en la que se establecen los temas a tratar, las cuestiones centrales de la investigación, la localización de los entrevistados, así como la confirmación de las condiciones de la misma.

Se ha utilizado una táctica directiva para obtener una información determinada y precisa, referente al problema planteado, evitando así posibles divagaciones. Para su registro se usa la grabación en audio, posteriormente se procede a la transcripción, reducción de datos e interpretación de elementos significativos mediante el programa AQAD-seis para Windows.

- Grupo de discusión con los técnicos para conocer la adecuación de la oferta formativa a las necesidades de la zona y a los NYE optamos por el grupo de discusión como estrategia especialmente útil para el análisis de situaciones que revisten complejidad, pudiendo aplicarse en diferentes momentos del proceso de investigación dependiendo de los objetivos perseguidos, teniendo como referencia a Ibáñez (2003) y Krueger (1991) por su especial relevancia en la contribución a la fundamentación teórica y encuadre metodológico del grupo de discusión.

La selección de las personas que van a componer el grupo de discusión es de gran trascendencia para el buen desarrollo del mismo. El grupo de técnicos de empleo responde a unos criterios mínimos de homogeneidad y heterogeneidad. La homogeneidad viene determinada por los objetivos del estudio, ya que es de suma importancia contar con ciertas características comunes (edad, experiencia profesional, formación académica, etc.) para que el grupo pueda construir unas señas de identidad. La heterogeneidad, por su parte, añade información a la discusión, aumenta la amplitud de perspectivas y posiciones y aporta mayores posibilidades a la construcción del material discursivo en el grupo. 


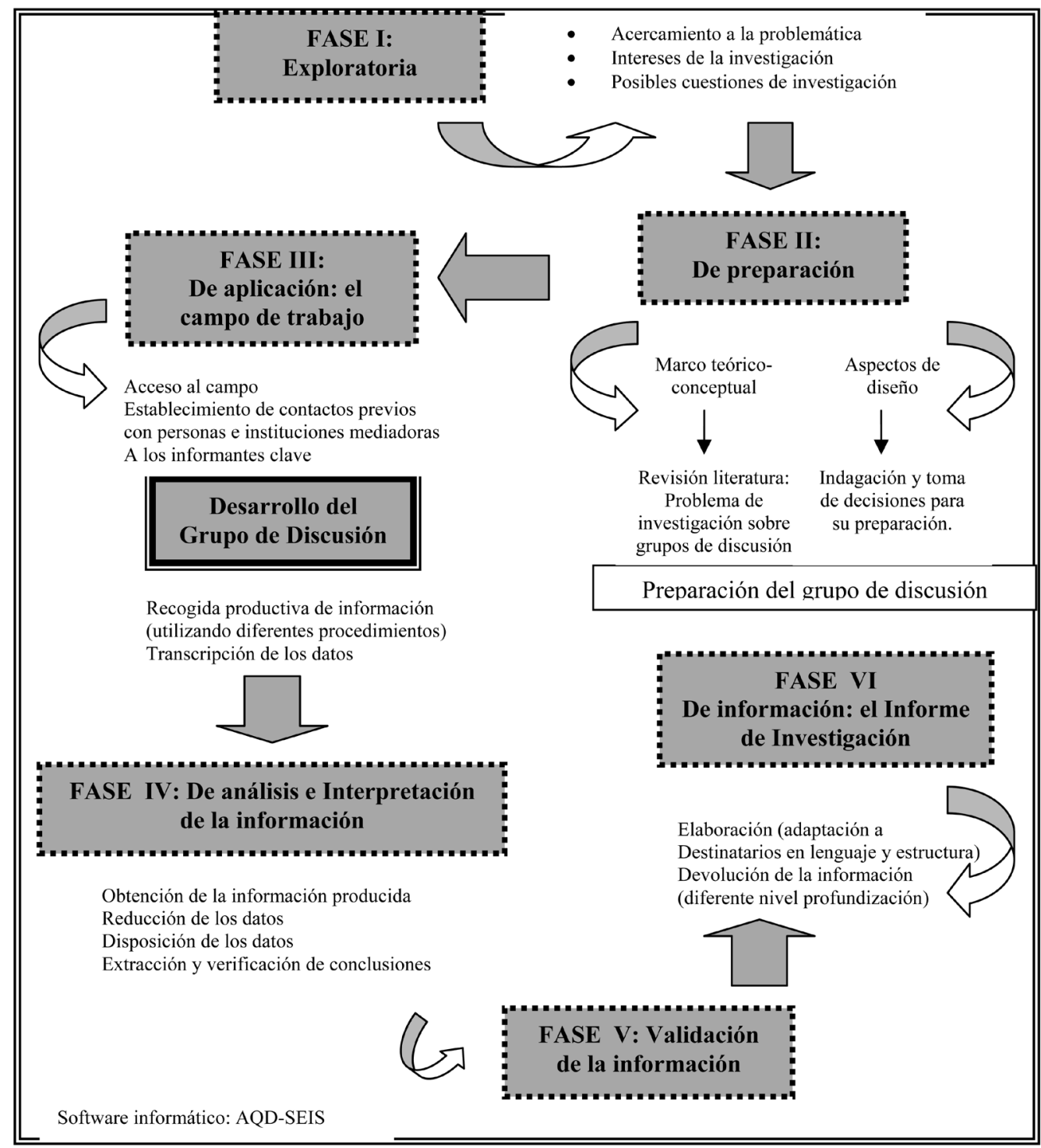

FIGURA 2

PROCESO SEGUIDO EN LOS GRUPOS DE DISCUSIÓN

El proceso seguido en lo grupos de discusión es el propuesto por Suárez (2005) y que exponemos a continuación por la claridad del mismo.

El grupo de discusión estuvo compuesto por 6 sujetos, los Agentes Locales de Promoción de Empleo de la estructura complementaria de la UTEDLT de Alhama de Granada. 


\subsection{Descripción de la muestra de estudio}

Población y muestra coinciden. En este estudio nos hemos centrado en la evaluación de las acciones formativas realizadas durante el año 2007 en la Comarca de Alhama de Granada, en su mayoría gestionadas por ALPEs, estas han sido:

- El Taller de Empleo de Arenas del Rey "Nueva Bermejales", compuesto por los módulos de "Albañilería" e "Instalaciones".

- El Taller de Empleo de Jayena "Turillas", integrado por el módulo de Turismo Rural y de Auxiliar de Ayuda a Domicilio.

- La Escuela Taller de Alhama de Granada "El Silo", constituido por los módulos de Carpintería de Madera y de Albañilería.

- El curso de F.P.O. de "Técnico en Información Turística" impartido por el Centro de Formación "Taxus Baccata" de Alhama de Granada.

De acuerdo a los planteamientos iniciales de este estudio, fundamentadas en las orientaciones de la Unión Europea sobre planes de empleo, se presta una especial atención a las informaciones referidas a los colectivos con especiales dificultades de inserción en la zona que se concretan en: mujeres, inmigrantes, jóvenes y personas con diversidad funcional.

Como podemos comprobar en la tabla $\mathrm{n}^{\circ} 3$, para la fase de exploración del contexto y el conocimiento de la situación socioeconómica y cultural de este territorio se realiza un análisis documental de informes y estudios previos, recopilando la información de distintos organismos e instituciones. En el resto de las fases de la evaluación se ha realizado un proceso de selección muestral intencional, seleccionando a los sujetos que pueden realizar una mejor aportación al objeto de estudio, considerando, cuando ha sido posible, la totalidad de los sujetos. La muestra quedo formada por los siguientes colectivos:

- Técnicos. La selección de los "informantes clave" se realizó en función de sus características profesionales y de las funciones que estos desempeñan en su trabajo. Deberían tener buen conocimiento del contexto de diferentes municipios de la Comarca, así como la realización de actividades relacionadas con la formación, el empleo y la integración social de colectivos desfavorecidos.

Estas condiciones las reunieron los ALPEs de la comarca estudiada y fueron ellos los que nos facilitaron la primera información requerida. De los seis que participaron en el estudio cinco son titulados superiores (Ingeniería Agrónoma, Ciencias Económicas, Pedagogía, Psicología y Ciencias Ambientales) y uno titulado medio en Relaciones Laborales. El 50\% son de género femenino y el 50\% de género masculino, con una media de edad de 35,3 años.

- Gestores. El 75\% de los directores de los programas formativos son mujeres y el $25 \%$ son de género masculino.

- Beneficiarios. Como se puede observar en la tabla $n^{\circ} 3$ la muestra productora de datos, en el cuestionario, esta compuesta por 61 personas con una media de edad $=$ 31,25 años, de los cuales el 83\% son de género femenino y el 17\% son hombres. El 73\% del alumnado tiene estudios básicos, el 24\% posee estudios de Bachillerato o Formación Profesional y tan solo un 3\% tiene titulación universitaria, por lo que se trata de un grupo de personas con un bajo nivel formativo. Por otra parte, un $79 \%$ del alumnado 


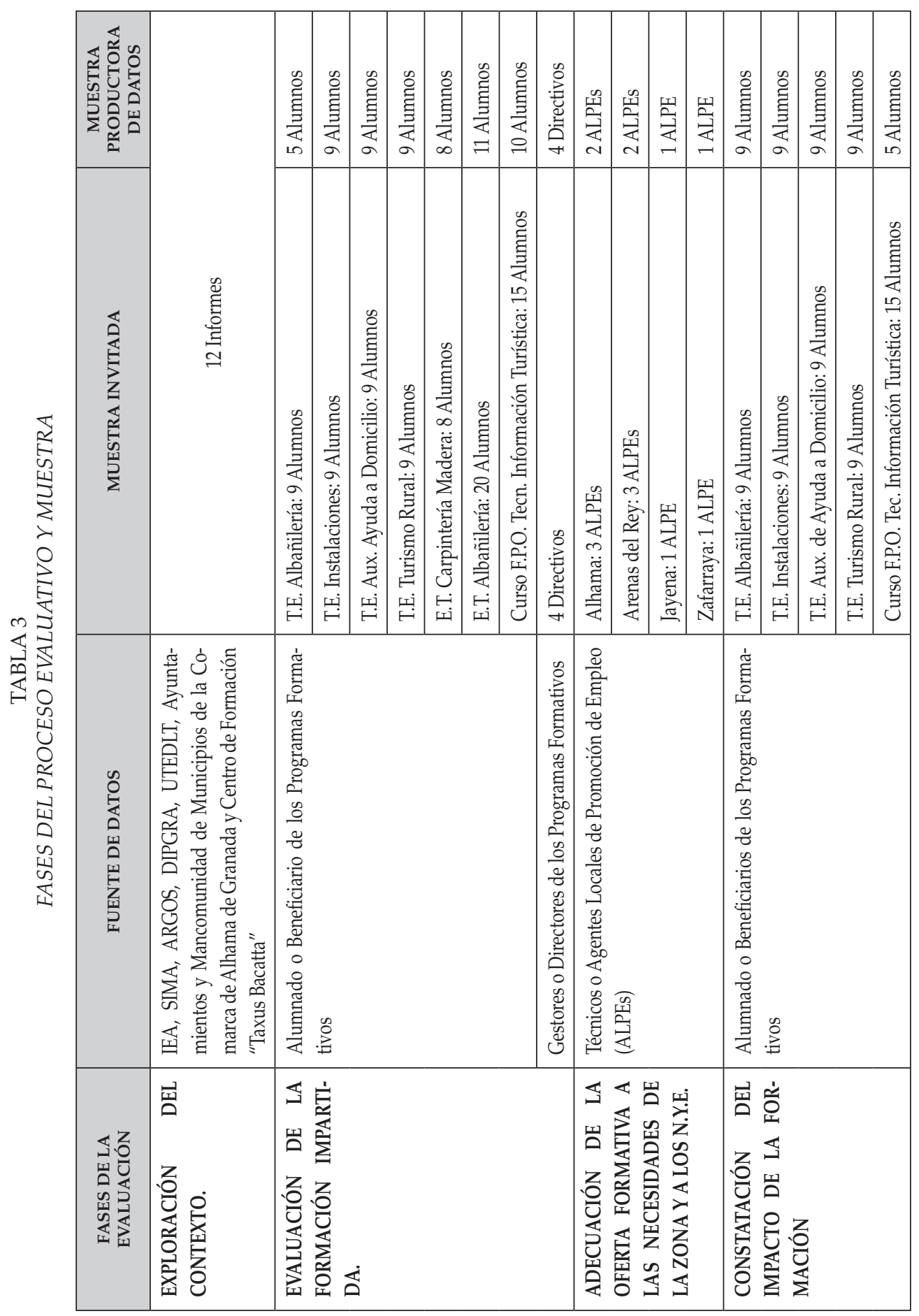


no posee experiencia laboral previa en el ámbito para el que están recibiendo formación y un $48 \%$ no ha realizado con anterioridad ningún curso de estas características.

\subsection{Técnicas de análisis de datos}

\section{A. Análisis documental}

Esta técnica se aplica en diversas parcelas educativas, fundamentalmente en la evaluación de programas formativos, nosotros lo hemos utilizado como apoyo a otros instrumentos de recogida de datos (grupo de discusión, entrevista y cuestionario), así como para validar la información obtenida.

Hemos utilizado documentos oficiales que podemos catalogar en material interno y externo. Los documentos internos son aquellos que circulan dentro de los Ayuntamientos de los Municipios donde se enmarca el estudio, la Mancomunidad de Municipios de la Comarca y la UTEDLT de Alhama de Granada. Los documentos externos utilizados han sido materiales producidos por las organizaciones para su comunicación con elementos externos: Instituto de Estadística de Andalucía (IEA), Sistema de Información Multiteritorial de Andalucía (SIMA), observatorio ARGOS, etc.

\section{B. Análisis descriptivo}

Para el análisis descriptivo de los datos obtenidos a través del cuestionario de evaluación se han agrupado los cursos impartidos en las distintas acciones formativas por bloques temáticos, quedando de la siguiente manera: Albañilería que agrupa los módulos impartidos en la E.T. "El Silo" y el T.E. "Nueva Bermejales"; los cursos de Turismo agrupan el módulo de Turismo del T.E. "Turillas" y el curso de "Técnico en información turística" impartido por un centro privado; el módulo de Auxiliar de ayuda a domicilio impartido por el T.E. "Turillas"; el módulo de Carpintería de madera impartido por la E.T. “El Silo” y el módulo de Instalaciones impartido por el T.E. "Nueva Bermejales". Los datos han sido analizados con el paquete estadístico SPSS 15.0, en función de las 3 dimensiones, 20 categorías y 80 ítems de las que se compone el cuestionario, estas son:

\section{Análisis de contenido}

Una vez recogidos los datos, a través del grupo de discusión y de las entrevistas explicitamos la finalidad del análisis para pasar a la exploración posterior de los mismos mediante la triangulación y así completar aquellos que consideremos incompletos con el fin de elaborar categorías. Se realiza una selección secuencial mediante un procedimiento abierto, con el que a medida que avanza la investigación se determinan nuevos conjuntos de fenómenos para el análisis. En primer lugar procedemos al análisis de los mismos, lo que en palabras de Huber y Marcelo (1990) supone estructurar y exponer unidades, para que, podamos extraer y confirmar conclusiones comprensivas.

Posteriormente se elaborarán unos códigos para la reducción de datos, es decir, se asignan abreviaciones o símbolos a unas frases o párrafos de las respuestas, en función 
TABLA 4

DIMENSIONES, INDICADORES E ÍTEMS DEL CUESTIONARIO AL ALUMNADO

\begin{tabular}{|c|c|c|}
\hline DIMENSIONES & INDICADORES & ÍTEMS \\
\hline \multirow{10}{*}{$\begin{array}{l}\text { 1. INFORMACIÓN } \\
\text { RELATIVA AL CURSO. }\end{array}$} & Objetivos del curso. & 3 ítems. \\
\hline & Contenidos del curso. & 4 ítems. \\
\hline & Metodología. & 4 ítems. \\
\hline & Actividades realizadas. & 3 ítems. \\
\hline & Organización del tiempo. & 3 ítems. \\
\hline & $\begin{array}{l}\text { Integración de los recursos que ofrece el } \\
\text { medio/contexto }\end{array}$ & 2 ítems. \\
\hline & Preocupación por la integración laboral. & 3 ítems. \\
\hline & Información facilitada. & 6 ítems. \\
\hline & Cantidad y calidad del material recibido. & 5 ítems. \\
\hline & Módulos transversales & 6 ítems. \\
\hline \multirow{3}{*}{$\begin{array}{l}\text { 2. INFORMACIÓN } \\
\text { RELATIVA AL ESPACIO, } \\
\text { MATERIAL EMPLEADO, } \\
\text { RECURSOS E } \\
\text { INSTALACIONES. }\end{array}$} & $\begin{array}{l}\text { Condiciones físicas del aula (espacio, } \\
\text { iluminación, temperatura,...). }\end{array}$ & 2 ítems. \\
\hline & Instalaciones. & 3 ítems. \\
\hline & Equipos y medios disponibles. & 3 ítems. \\
\hline \multirow{7}{*}{$\begin{array}{l}\text { 3. INFORMACIÓN } \\
\text { RELATIVA AL } \\
\text { FORMADOR. }\end{array}$} & Cualidades personales. & 5 ítems. \\
\hline & Competencias para enseñar. & 7 ítems. \\
\hline & Ambiente y clima de clase. & 4 ítems. \\
\hline & Relación con los alumnos. & 3 ítems. \\
\hline & Innovación educativa. & 4 ítems. \\
\hline & Evaluación escolar. & 7 ítems. \\
\hline & Orientación sobre el empleo. & 3 ítems. \\
\hline
\end{tabular}

de los objetivos de nuestra investigación. Para el análisis de los datos cualitativos, a través de un proceso inductivo-deductivo, hemos elaborado un sistema de categorías compuesto por 4 dimensiones, 10 categorías y 57 rasgos y han sido analizados con el programa AQUAD 6.0. 
TABLA 5

UNIDADES DE REGISTRO INCLUIDAS DENTRO DE LAS CATEGORÍAS

\begin{tabular}{|c|c|c|}
\hline DIMENSIONES & CATEGORÍAS & cóDIGOS \\
\hline \multirow{5}{*}{$\begin{array}{l}\text { CURSOS DE F.P.E. } \\
\text { REALIZADOS }\end{array}$} & CAL: ALUMNADO & $\begin{array}{l}\text { CAL: Motivación del alumnado. } \\
\text { CAL: Selección del alumnado. } \\
\text { CAL: Características beneficiarios. }\end{array}$ \\
\hline & CCO: CONTENIDOS & $\begin{array}{l}\text { CCO: Adaptación a los conocimientos. } \\
\text { CCO: Coherencia. } \\
\text { CCO: Cursos y módulos impartidos. } \\
\text { CCO: Secuenciación teoría/ práctica. }\end{array}$ \\
\hline & CGE: GESTIÓN & $\begin{array}{l}\text { CGE: Actividades vinculadas sociedad. } \\
\text { CGE: Adecuación de los recursos. } \\
\text { CGE: Coordinación y comunicación } \\
\text { CGE: Organización. }\end{array}$ \\
\hline & COB: OBJETIVOS & $\begin{array}{l}\text { COB: Coherencia. } \\
\text { COB: Grado de consecución. } \\
\text { COB: Situacionalidad. }\end{array}$ \\
\hline & CPE: PERSONAL DOCENTE & $\begin{array}{l}\text { CPE: Características del docente. } \\
\text { CPE: Formación pedagógica. } \\
\text { CPE: Formación técnica. } \\
\text { CPE: Libertad docente. } \\
\text { CPE: Selección del docente. }\end{array}$ \\
\hline \multirow{4}{*}{$\begin{array}{l}\text { DISEÑO DE } \\
\text { LAS ACCIONES } \\
\text { FORMATIVAS }\end{array}$} & DCO: CONTEXTO & $\begin{array}{l}\text { DCO: Colectivos en riesgo de exclusión. } \\
\text { DCO: Cultura emprendedora. } \\
\text { DCO: Potencial endógeno. } \\
\text { DCO: Tejido empresarial. } \\
\text { DCO: Contexto estratégico. }\end{array}$ \\
\hline & DFO: FORMACIÓN OFERTADA & $\begin{array}{l}\text { DFO: Cursos ofertados. } \\
\text { DFO: Dispositivos de F.P. } \\
\text { DFO: Características beneficiarios. } \\
\text { DFO: Entidades que ofertan formación. } \\
\text { DFO: Seguimiento del programa. }\end{array}$ \\
\hline & $\begin{array}{l}\text { DNU: NUEVAS NECESIDADES } \\
\text { FORMATIVAS }\end{array}$ & $\begin{array}{l}\text { DNU: Análisis de necesidades. } \\
\text { DNU: Características beneficiarios. } \\
\text { DNU: Formación nuevas necesidades. }\end{array}$ \\
\hline & DPL: PLANIFICACIÓN & $\begin{array}{l}\text { DPL: Aportaciones políticas. } \\
\text { DPL: Aportaciones técnicas. } \\
\text { DPL: Características beneficiarios. } \\
\text { DPL: Normas básicas de organización. } \\
\text { DPL: Recursos disponibles. }\end{array}$ \\
\hline
\end{tabular}




\begin{tabular}{|c|c|c|}
\hline DIMENSIONES & CATEGORÍAS & CÓDIGOS \\
\hline \multirow[b]{2}{*}{ IMPACTO } & IEF: RESULTADOS EFECTOS & $\begin{array}{l}\text { IEF: Intención de inserción. } \\
\text { IEF: Interés de los empleadores. } \\
\text { IEF: Posibilidades de inserción. }\end{array}$ \\
\hline & IPR: RESULTADOS-PRODUCTOS & $\begin{array}{l}\text { IPR: Creación de empleo. } \\
\text { IPR: Competencias profesionales } \\
\text { IPR: Nuevas acciones formativas de- } \\
\text { mandadas. } \\
\text { IPR: Resultados en el ámbito formativo. } \\
\text { IPR: Resultados en el ámbito laboral. } \\
\text { IPR: Resultados en el ámbito personal.. } \\
\text { IPR: Satisfacción. }\end{array}$ \\
\hline \multirow{5}{*}{$\begin{array}{l}\text { NUEVOS } \\
\text { YACIMIENTOS DE } \\
\text { EMPLEO }\end{array}$} & $\begin{array}{l}\text { NAM: SERVICIOS DE MEDIO } \\
\text { AMBIENTE }\end{array}$ & NAM: Control de la energía. \\
\hline & $\begin{array}{l}\text { NCU: SERVICIOS CULTURALES Y } \\
\text { DE OCIO }\end{array}$ & $\begin{array}{l}\text { NCU: Forma física y deportes de } \\
\text { aventura. } \\
\text { NCU: Nuevas formas de turismo. }\end{array}$ \\
\hline & $\begin{array}{l}\text { NME: MEJORAS EN EL MARCO } \\
\text { DE LA VIDA }\end{array}$ & $\begin{array}{l}\text { NME: Renovación de inmuebles. } \\
\text { NME: Transportes colectivos. }\end{array}$ \\
\hline & $\begin{array}{l}\text { NTR: ACTIVIDADES } \\
\text { TRADICIONALES }\end{array}$ & $\begin{array}{l}\text { NTR: Artesanía. } \\
\text { NTR: Sector agrario. } \\
\text { NTR: Sector servicios. }\end{array}$ \\
\hline & $\begin{array}{l}\text { NVI: SERVICIOS DE LA VIDA } \\
\text { DIARIA }\end{array}$ & $\begin{array}{l}\text { NVI: Atención a la tercera edad. } \\
\text { NVI: Nuevas tecnologías. }\end{array}$ \\
\hline
\end{tabular}

\section{RESULTADOS}

Por no hacernos muy extensos vamos a exponer los resultados más destacados de este trabajo en función del tipo de análisis de datos realizado.

Mediante el análisis descriptivo del cuestionario al alumnado podemos observar que las acciones formativas están bien valoradas, con una puntuación media de 4,44 en una escala del 1 al 6, siendo "Información relativa al curso" la dimensión que mayor puntuación alcanza con un valor medio de 4,60, seguido de la dimensión "Información relativa al espacio, material empleado, recursos e instalaciones" con una valoración de 4,39 y la dimensión "Información relativa al formador" valorada en 4,35.

A través del análisis de contenido de la entrevista a los directivos, del grupo de discusión con los técnicos de empleo y al alumnado conseguimos los siguientes resultados:

Los valores de las dimensiones en la entrevista a los directores son, por orden decreciente: "Cursos de F.P.E. realizados" (57\%), "Diseño de las acciones formativas" (20\%), "Impacto" (17\%) y "NYE" contemplados como posibles nichos de inserción laboral con un $6 \%$. 
Los valores de las dimensiones, por orden decreciente, del grupo de discusión con los técnicos de empleo son: "Diseño de las acciones formativas" (54\%), seguido de "Cursos de F.P.E. realizados" (24\%), "NYE" (14\%) e "Impacto" (8\%).

La dimensión más valorada en la entrevista telefónica al alumnado ha sido "Impacto" (66\%), seguida de "Diseño de las acciones formativas" (12\%), "Cursos de FPE realizados" (11\%) y "NYE" (11\%).

\section{CONCLUSIONES}

Destacamos a continuación algunas de las conclusiones más relevantes en función de cada uno de los cuatro objetivos planteados:

Primer objetivo "Analizar las necesidades de FPE en la Comarca de Alhama de Granada":

- Si se contrastan los datos recabados relativos a la formación ofrecida en el territorio y a las necesidades detectadas, se observa cómo ha habido una inadecuación de las enseñanzas impartidas con las características del mercado laboral de ámbito comarcal, especialmente debido a dos cuestiones principales: por una acusada limitación de recursos (reducida oferta formativa y escasa variedad), y por el mantenimiento de los mismos módulos de formación reglada (FP de Automoción y de Auxiliar Administrativo) formativos sin adecuarse a las nuevas realidades.

- Se demanda mayor flexibilidad por parte de la Administración respecto al número de alumnos requeridos para la implementación de las acciones formativas, ya que la escasez de habitantes de la mayoría de los municipios dificulta la realización de dichos cursos. De forma general se considera que a pesar de tratarse de una zona rica en recursos naturales y con grandes posibilidades de expansión en el ámbito agrario y turístico, carece de tejido empresarial para la inserción del alumnado, por lo que se debería incidir en el autoempleo y el acompañamiento.

Segundo objetivo "Evaluar los cursos desarrollados y al profesorado que los ha impartido":

- En general el alumnado está bastante satisfecho con la formación recibida. Siendo una nota preocupante que el ítem que menor puntuación alcanza es el referente a la "Preocupación por la inserción laboral", con un valor medio = 3,3, teniendo en cuenta que es el objetivo prioritario que persiguen estas acciones formativas.

- Todas las acciones formativas desarrolladas durante el transcurso de este estudio han ido dirigidas prioritariamente a colectivos en riesgo de exclusión social, siendo el alumnado beneficiario de estas principalmente mujeres.

Tercer objetivo: Realizar un estudio comparativo entre las necesidades de FPE, la formación ofertada y el impacto de ésta sobre la realidad socioeconómica de la Comarca de Alhama:

- La selección del alumnado, según los ALPES, no ha sido la adecuada. Pues a pesar del interés por la formación que muestra el alumnado, no tienen intención real de inserción en el mercado laboral, ya que su principal motivación es la remuneración económica, perpetuando así el desarrollo de una perspectiva subsidiaria y escasamente emprendedora. 
- Por otra parte consideran que la oferta formativa es reiterativa: informática, ingles, albañilería e instalaciones, con escasas posibilidades de inserción del alumnado, especialmente en lo que al módulo de albañilería se refiere, ya que se encuentra con la dificultad añadida de que la mayoría del alumnado son mujeres, socialmente poco consideradas para su contratación en este sector.

- El impacto varía en función de las distintas acciones formativas. El alumnado tiene posibilidades reales de inserción, especialmente si optan por el autoempleo. La inserción en el mercado laboral de las alumnas del módulo de turismo han sido bajos, en el módulo de Ayuda a domicilio han sido elevados, mientras que en el de albañilería e instalaciones parecen diferencias en función del género. El alumnado está satisfecho con la formación recibida en las diferentes cursos En todas las acciones formativas destaca la satisfacción del alumnado y el impacto producido principalmente en los ámbitos personal, formativo y laboral.

- Aunque se están realizando acciones formativas dirigidas al colectivo femenino y juvenil, resalta la necesidad de ampliar la oferta para la población inmigrante, principalmente cursos de español. Así como para el colectivo de jóvenes pues la oferta de formación profesional reglada es muy limitada, siendo preciso orientar las dichas acciones hacia actividades emergentes. La población de personas con discapacidad no es significativo para desarrollar acciones formativas específicas destinadas a este colectivo.

Cuarto objetivo "Identificar los NYE en la Comarca de Alhama de Granada para el establecimiento de nuevos procesos formativos:

- Actividades tradicionales: Artesanía, Sector agrario (viverismo, poda, agricultura ecológica) y Sector servicios (hostelería, comercio, administración, contabilidad e informática).

- Servicios culturales y de ocio: Nuevas formas de turismo (natural, rural, guía, relax, visitas guiadas), Forma física y deportes de aventura (actividades de ocio y tiempo libre en contacto con la naturaleza: rapel, piragüismo, tiro con arco, descenso de barrancos, etc) y animación socio-cultural.

- Servicios de medio ambiente: Energías renovables (biomasa e instalación de placas solares) y cuidado del medioambiente.

- Mejoras en el marco de la vida: renovación y rehabilitación de viviendas para la promoción y desarrollo del turismo rural, transportes y telecomunicaciones.

- Servicios de la vida diaria: Atención a la tercera edad, cuidado de los niños y limpieza.

Por último, en el informe final del estudio se realizaron una serie de propuestas orientadas a la intervención y mejora para la integración de los nuevos empleos en el tejido socio-laboral. Igualmente se sugirió el diseño e implementación de nuevas líneas de intervención formativa adaptadas a los NYE, mediante el planteamiento de líneas estratégicas basadas en los recursos endógenos. Se insiste en la necesidad de una estrategia planificada de desarrollo local a nivel técnico, en la que teniendo en cuenta las aportaciones políticas y las demandas de la ciudadanía se acometa un proyecto consensuado para el desarrollo de toda la zona. 


\section{REFERENCIAS BIBLIOGRÁFICAS}

Albrecht, D.E; Mulford, C. et Albrecht, S.L. (2000). Poverty in Nonmetropolitan America: Impacts of Industrial, Employment, and family Structure Variables. En Rural Sociology, 65(1), 87-103.

Berrocal, E. y Buendía, L. (2003). Evaluación de los cursos de FPO en la Ciudad Autónoma de Ceuta. Comunicación en el XI Congreso de Modelos de Investigación Educativa: Investigación y Sociedad, celebrado en Granada. Septiembre.

Biencinto, C. y Carballo, R. (2004). Revisión de modelos de evaluación del impacto de la formación en el ámbito sanitario: de lo general a lo específico. RELIEVE, 10(2), 101-116.

Buendía, L. y Berrocal, E. (2008). “Evaluación de un programa de formación ocupacional para la inserción laboral". RIE, 26(1), 157-190.

Chiva, I. (2006). Evaluación de los programas de formación ocupacional para el colectivo de mujeres. RELIEVE, 12(1), 49-74.

Comisión de las Comunidades Europeas. (1993). Crecimiento, competitividad y empleo. Retos y pistas para entrar en el siglo XXI. Libro Blanco. Bruselas/Luxemburgo: Oficina de Publicaciones de las Comunidades Europeas.

De Miguel, M; Pereira, M.; Pascual, J. y otros. (2008): Evaluación de programas de empleo-formación. Metodología de estudio. RIE, 26(1), 157-190.

Expósito, J.; Olmedo, E. y Fernández, A. (2004): Patrones metodológicos en la investigación española sobre evaluación de programas educativos. RELIEVE, 10(2), 1-24.

Folgueiras, P. y Marín, M.A. (2009): Evaluación del proyecto de mejora de la calidad educativa mediante la incorporación del enfoque de género en escuelas de la República Dominicana: resultados de su impacto. RIE, 27(2), 487-508.

García, B. (1999). La sociedad rural ante el siglo XX. Madrid: MAPA.

García, B. e Izcara, S.P. (2000). Pluriactividad y diversificación de ingresos en el medio rural español. En Sociología del Trabajo, 38, 119-134.

Huber, G. y Marcelo, C. (1990). Algo más que recuperar palabras y contar referencias. La ayuda del ordenador en el análisis datos cualitativos. Revista de Enseñanza, 8, 69-83.

Ibáñez, J. (2003). Cómo se realiza una investigación mediante grupos de discusión. En M. García Ferrando; J. Ibáñez y F. Alvira (Eds.). El análisis de la realidad social. Métodos y técnicas de Investigación Social. Madrid: Alianza.

IEA: Instituto de Estadística de Andalucía (2007). Sistema de información multiterritorial de Andalucía. En http://www.juntadeandalucia.es/institutodeestadistica/ sima/htm/sm23092.htm. (Consultado el 27 de noviembre de 2007).

Krueger, R.A. (1991). El grupo de discusión. Guía Práctica para la investigación aplicada. Madrid: Pirámide.

Manzanares, A (2006). La formación para el trabajo y la mujer en contextos rurales: el proyecto IMUMEL. En Educar, 38, 171-192.

Perales, M.J; Jornet, J. y Suarez, J. (2000) La evaluación de la formación ocupacional y continua. RIE, 18(2), 521-53

Pérez Juste, R. (2000). La evaluación de programas educativos: conceptos básicos, planteamientos generales y Problemática. RIE, 18(2), 261-287. 
Stake, R.E. (1976). A theoretical stament of responsive evaluation. Studies in Educational Evaluation, 2, 19-22.

Stufflebeam, D.L. y Shinkfield, A. J. (1987). Evaluación sistemática. Guía teórica y práctica. Barcelona: Paidos/MEC.

Suárez, M. (2005). El grupo de discusión. Una herramienta para la investigación cualitativa. Barcelona: Laertes.

Tejáda, J.; Ferrández, E., y otros. (2008). Implicaciones de la evaluación de impacto. Una experiencia en un Programa de Formación de Formadores. Bordón. Revista de Pedagogía, 60(1), 163-186.

Yaus, D.A. (1990): Surveys in Social Research. Londres: Unwin Hyman.

Fecha de recepción: 23 de enero de 2011.

Fecha de revisión: 05 de febrero de 2011.

Fecha de aceptación: 20 de marzo de 2011. 
